\title{
Sexual precocity: sex incidence and aetiology
}

\author{
N A Bridges, J A Christopher, P C Hindmarsh, C G D Brook
}

\begin{abstract}
The aetiology of 197 girls and 16 boys presenting with sexual precocity was reviewed. Ninety one girls and four boys had central precocious puberty (M:F 23:1); a cause was identified in all the boys but in only six girls. All boys with precocious puberty need detailed investigation; in girls investigation should be based on clinical findings, particularly the consonance of puberty.

(Arch Dis Child 1994; 70: 116-118)
\end{abstract}

The introduction of a range of non-invasive imaging techniques in recent years has made the existing literature, which was based on observation, outdated. New imaging techniques, such as computed tomography, magnetic resonance imaging, and pelvic ultrasound and new therapeutic regimens (gonadotrophin releasing hormone (GnRH) analogues) have prompted a reappraisal of the diagnostic classification of sexual precocity. We have reviewed our experience of the differential diagnosis of this condition at the Middlesex Hospital over a 15 year period.

\section{Patients and methods}

A total of 213 children presented with a diagnosis of sexual precocity between 1975 and 1990. Sexual precocity was defined as the appearance of secondary sexual characteristics before the age of 8 years in girls and 9 years in boys. All the children were evaluated clinically using standard anthropometric measures and puberty was staged according to the method of Tanner. ${ }^{1}$ Ultrasound examination of the ovary and uterus was carried out in girls; recordings of ovarian and uterine volume and estimates of ovarian morphology (follicular size and number) were made.

Endocrine investigations included 24 hour urinary steroid profiles, thyroid function tests, GnRH stimulation tests and, more recently, 24 hour blood sampling at 20 minute intervals for the estimation of serum luteinising hormone (LH), follicle stimulating hormone (FSH), and testosterone or oestradiol concentrations. Recently magnetic resonance imaging has superseded computed tomography as the preferred cerebral imaging technique. \section{Cobbold Laboratories,}

Endocrine Unit, Middlesex Hospital, Mortimer Street, London W1N 8AA N A Bridges

J A Christopher PC Hindmarsh C G D Brook

Correspondence to: Professor Brook.

Accepted 14 September 1993

\section{Results}

Of 213 children presenting with sexual precocity 197 were girls and 16 boys. Patients were grouped into diagnostic categories based on a combination of clinical assessment, pelvic ultrasonography. The figure illustrates the distribution of these categories in the two sexes.

\section{(1) GONADOTROPHIN DEPENDENT PRECOCIOUS} PUBERTY

\section{(A) Central precocious puberty}

This was defined as the appearance of secondary sexual characteristics in a normal sequence associated with early, but otherwise normal, activation of the hypothalamo-pituitary-gonadal axis. Endocrine studies revealed a dominance of $\mathrm{LH}$ over FSH. Stimulation with $\mathrm{GnRH}$ in these children resulted in a response similar to that seen in normally timed puberty. Ninety one girls presented with central precocious puberty and 85 were idiopathic. The remaining six had central precocious puberty secondary to previously diagnosed intracranial pathology (two isolated hydrocephalus, two brain tumours, one hypothalamic hamartoma, and one arachnoid cyst). Precocious puberty was not the presenting symptom of the intracranial pathology in any of the girls. Four boys presented with central precocious puberty and a cause was found in all (one brain tumour, one hypothalamic hamartoma, one after severe head trauma, and one congenital adrenal hyperplasia due to 21-hydroxylase deficiency).

\section{(B) Sexual precocity associated with raised gonadotrophins}

This condition was distinguished from central precocious puberty, and was either associated with isolated breast development (premature thelarche) where serum FSH concentrations predominated and pelvic ultrasonography usually demonstrated a single cyst in one ovary, or with primary hypothyroidism where serum FSH concentrations again predominated. Growth rate was normal in girls with premature thelarche and there was no advance of skeletal maturation.

\section{(C) Thelarche variant}

This condition appears to be intermediate between premature thelarche and central precocious puberty. Serum LH concentrations were not as raised as in central precocious puberty and although there were signs of pubertal development, neither growth rate nor skeletal maturation were accelerated. ${ }^{2}$ Twenty girls had this condition. endocrine evaluation, radiological imaging, and
(2) GONADOTROPHIN INDEPENDENT PRECOCIOUS PUBERTY (GIPP)

This was defined as sexual precocity without hypothalamo-pituitary-gonadal activation. This 

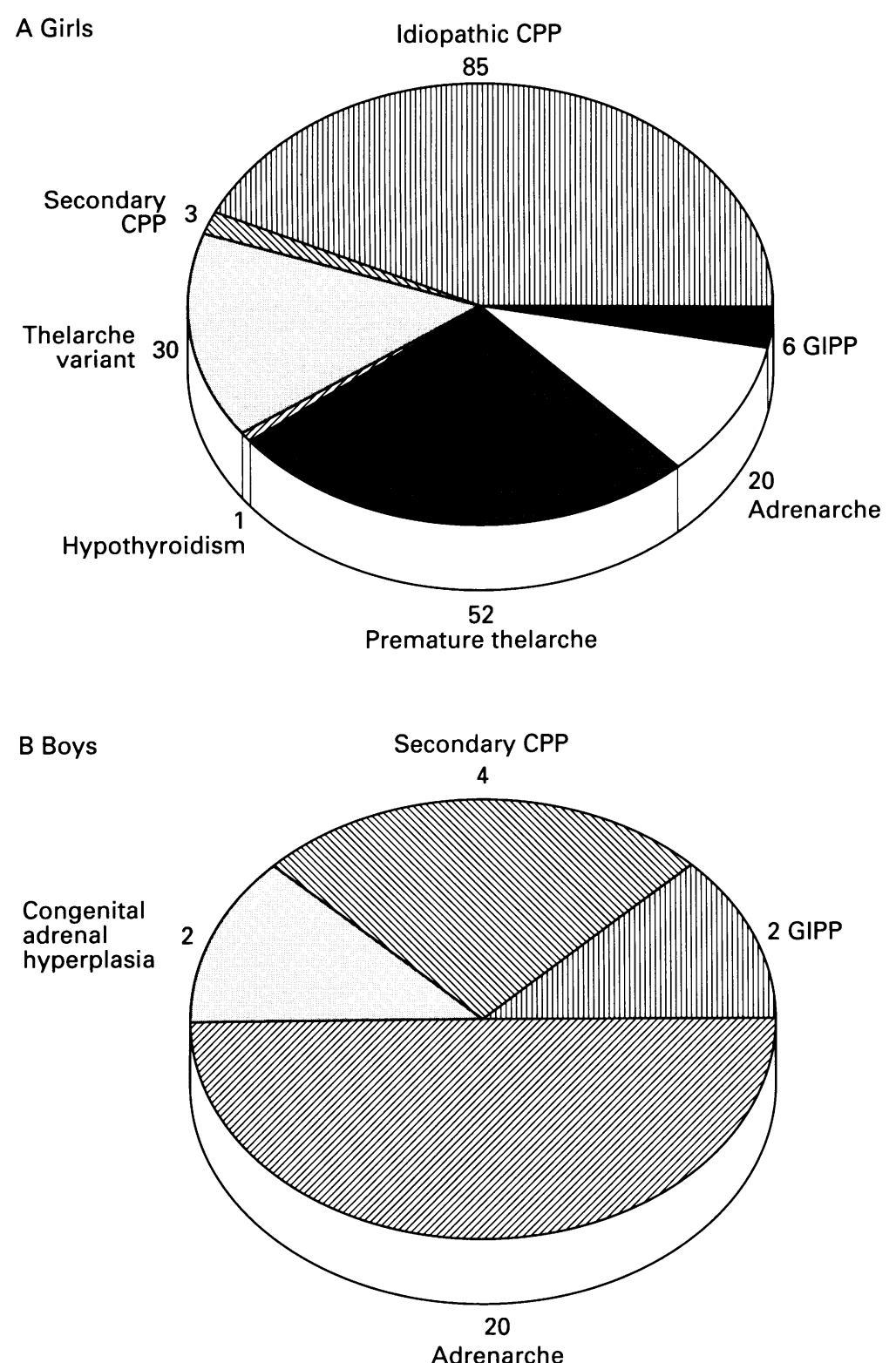

Pie charts showing numbers of children presenting with sexual precocity with each diagnosis in $(A)$ girls and $(B)$ boys; $C P P=$ central precocious puberty.

condition was diagnosed using defined criteria. GIPP was an unusual cause of sexual precocity in girls, all three of whom had the McCuneAlbright syndrome. ${ }^{34}$ Two boys had GIPP. Stimulation with $\mathrm{GnRH}$ in these individuals reveals low baseline $\mathrm{LH}$ and $\mathrm{FSH}$ concentrations and an absent response (suppressed by the pubertal levels of sex steroids).

(3) INCREASED ANDROGEN PRODUCTION ONLY Manifestations of adrenarche (isolated pubic and axillary hair development) was a common diagnosis in both boys (50\%) and girls (15\%). Undertreated congenital adrenal hyperplasia (11 $\beta$ hydroxylase deficiency) was a cause in two boys.

\section{Discussion}

These data demonstrate that central precocious puberty presents more commonly in girls than boys (female to male ratio 23:1). In the vast majority of girls with central precocious puberty no cause was found, whereas a cause was identified in all the boys. A possible explanation as to why idiopathic central precocious puberty is more common in girls is that activation of the hypothalamopituitary-gonadal axis is easier in girls. Boys with delayed puberty undergoing treatment with GnRH require higher amplitude pulses than girls to achieve similar pubertal development. ${ }^{5}$ Suppression of sexual precocity with a $\mathrm{GnRH}$ analogue is more difficult in girls than boys. ${ }^{6}$ Boys with delayed puberty outnumber girls by 20 to one.

During the 15 year period that we have examined, the technical developments in computed tomography and later magnetic resonance imaging have greatly improved imaging of the hypothalamus and pituitary. Magnetic resonance imaging is superior to computed tomography in imaging of the hypothalamus and pituitary. This raises the question as to whether small lesions such as hamartomas are being visualised now in children whose central precocious puberty would have been labelled as idiopathic in the past. All the girls with secondary central precocious puberty had some symptoms of their central lesion as well as those of central precocious puberty, and we found no girls in our series with small asymptomatic lesions. However, to find such lesions would require that all girls with central precocious puberty were scanned; this is not currently our policy and we feel it cannot be justified in terms of any improvement in the management of these patients.

Another imaging technique that has developed over the last 15 years is pelvic ultrasonography, which is a useful method of assessing sexual precocity in girls. At present, every girl with sexual precocity has a pelvic ultrasound. This change in practice has not affected our diagnostic criteria but has meant that fewer girls have blood testing. Attention needs to be paid in boys to pubertal staging, which may dictate the direction of endocrine investigation.

There have been parallel improvements over 15 years in gonadotrophin and sex steroid assays, but the assessment of gonadotrophin secretion remains difficult and may not add to the information gained by clinical assessment. The response to stimulation with $\mathrm{GnRH}$ is of value in distinguishing centrally mediated from gonadotrophin independent precocious puberty. The gonadotrophin pulsatility observed in premature thelarche and thelarche variant can be demonstrated by 24 hour sampling, but clinical assessment followed by the demonstration of a normal growth velocity and lack of pubertal progress are just as informative.

The importance of the consonance of puberty as described by Tanner ${ }^{1}$ cannot be over emphasised: any deviation from the regular smooth progression through puberty requires an explanation. Improvements in imaging and therapeutic options have allowed for a more dynamic classification of sexual precocity. Diagnostic classification has impor- 
tant implications in terms of targeting treatment regimens; for example, GnRH analogues should be used in central (gonadotrophin dependent) precocious puberty whereas blockers of sex steroid biosynthesis are more appropriate for the management of GIPP. Newer classifications have aided our understanding of the prognosis of sexual precocity. Central precocious puberty or GIPP leads to a reduced adult height whereas premature thelarche, thelarche variant, and premature adrenarche are associated with a normal height prognosis and do not require intervention.
1 Tanner JM. Growth at adolescence. 2nd Ed. Oxford: Blackwell Scientific Publications, 1962

2 Stanhope R, Brook CGD. Thelarche variant. Acta Endocrinol (Copenh) 1990; 123: 481-6.

3 Rosenthal SN, Grumbach MM, Kaplan SL. Gonadotrophin independent familial sexual precocity with premature Leydig and germinal cell maturation (familial testotoxicosis): effect of a potent luteinising hormone releasing factor agonist and medr agonist and medroxyprogesterone acetate therapy in fou docrinol Metab 1983; 57: 571-9.

Mauras N, Blizzard RM. The McCune-Albright syndrome. Acta Endocrinologica Scandinavica [Supplement] 1986; 279, 207-17.

5 Stanhope R, Brook CGD, Pringle PJ, Adams J. Induction of puberty by pulsatile gonadotrophin releasing hormone. Lancet 1987; ii: 552-5.

6 Donaldson MD, Stanhope R, Lee TJ, Price DA, Brook CG Savage DC. Gonadotrophin responses to gonadotrophin releasing hormone in precocious puberty treated with gonadotrophin releasing hormone analogue. Clin Endocrinol (Oxf) 1984; 21: 499-503. 